\title{
Photoinduced Changes in $\mathrm{As}_{2} \mathrm{~S}_{3}$ Thin Films
}

\author{
A. Abd-El Mongy \\ Physics department Faculty of Science, Helwan University, \\ Cairo, Egypt*
}

\begin{abstract}
Optoelectronic, photoelectric and dc conductivity of amorphous $\mathrm{As}_{2} \mathrm{~S}_{3}$ thin films prepared by thermal evaporation are investigated. Absorption coefficient as well as photoconductivity spectral dependence is used to estimate the band gap. The temperature dependence of $d c$ conductivity and steady state photoconductivity revealed a discontinuity at $150 \mathrm{~K}$. A comparative study of the film properties before and after irradiation demonstrated the light-induced changes. Photodarkening, with metastable and transient components, is observed. The photoconductivity kinetics indicated that the new localized states, created by illumination, dominate the recombination process in a similar manner to the original states. The similarity in behaviours obtained before and after irradiation suggests that photochemical changes are produced by illumination.
\end{abstract}

\section{Introduction:}

Essentially all amorphous semiconductors undergo changes in their physical properties when they are exposed to band gap light. In the stoichiometric arsenic chalcogenides, such as $\mathrm{As}_{2} \mathrm{~S}_{3}$, the arsenic atoms are bonded to three chalcogen atoms in a pyramidal configuration [1]. Arsenic trisulfide $\left(\mathrm{As}_{2} \mathrm{~S}_{3}\right)$ is a technically important class of materials because of its good transparency in the $0.7-11 \mu \mathrm{m}$ wavelength range. It also has a variety of applications in optical imaging, hologram recording, and electronic devices including electro-optic information storage devices and optical mass memories [2]. Various methods have been applied to deposit $\mathrm{As}_{2} \mathrm{~S}_{3}$ thin films with an optical band gaps ranged from 1.82 to $2.38 \mathrm{eV}$ and with resistivity in the range $10^{6}$ to $10^{9} \Omega \mathrm{cm}[3]$.

Email: abdohelwanegypt@yahoo.com 
The optical absorption in amorphous semiconductors still a matter of controversy, by comparison with that in crystalline which has been understood firmly based on band theory [4]. Then to get insights into optical absorption in amorphous materials, variety of features: Tauc gaps, Urbach edges, weak absorption tails, mobility edges and localized states have to be characterized [5\&6]. Both the model of Mott and Davis [7] and Tauc's model [8], derived under different assumptions, give the dependence of the fundamental absorption edge in most amorphous semiconductors.

Different models have been proposed for interpretation of the absorption edge [9\&10]. Recently the overall gap is assumed a function of two parameters the covalent bond length and the interlayer spacing [11]. Accordingly in the case of $\mathrm{As}_{2} \mathrm{~S}_{3}$ generally three types of covalent bonds can exist (As-S, As-As, S-S bonds) the distribution of which should depend on the method of the film preparation.

Photodarkening in arsenic chalcogenides, or the shift of the optical absorption edge to smaller energies with illumination has both scientific and technological importance [12-14]. Over the years many models have been proposed to explain this effect [15-18] and most explanations involve structural changes which in turn affect the electronic states. However, none has proved definite for all the materials in which it has been observed.

Photinduced changes have been reported as metastable, that is reversible on long time scales and by annealing. This has not been observed in crystalline materials nor does it occur in group IV or $\mathrm{V}$ amorphous semiconductors (e.g. a-Si). Moreover it has been observed that in amorphous germanium chalcogenides, illumination increases the band gap, a phenomenon known as photobleaching [19].

Despite this considerable interest, it seems not possible to draw general conclusions and still further studies are needed for revealing unknown nature in photoelectronic excitations of amorphous materials. The present paper deals with preparation and characterization of evaporated $\mathrm{As}_{2} \mathrm{~S}_{3}$ films. The film properties (optical absorption, electrical conductivity and photoconductive excitation spectra) are compared before and after irradiation. Photodarkening is also tested. 


\section{Experimental Details:}

\subsection{Film Preparation}

Films were prepared by thermal evaporation of high purity glassy arsenic trisulphide $\left(\mathrm{As}_{2} \mathrm{~S}_{3}\right)$ in a standard high -vacuum unit maintained at $5 \times 10^{-4} \mathrm{~Pa}$ residual pressure. A resistively- heated Tantalum crucible was used as an evaporation source which allows the sublimation and evaporation of $\mathrm{As}_{2} \mathrm{~S}_{3}$ without local overheating, thus preventing non-desirable effects of thermal decomposition. The substrates used were Corning 7059 glass plates, which exhibit a negligible absorption in the visible and near infrared regions. The deposition rate was $\sim 0.4-0.5 \mathrm{~nm} / \mathrm{s}$ at normal incidence and the substrate was kept at a temperature of $305 \mathrm{~K}$. The samples were annealed at the appropriate temperature $(443 \mathrm{~K})$ which is below the glass transition temperature for five hours in vacuum to eliminate the effects of heat and previous light exposure. The thickness and deposition rate of the growing films were measured using a quartz crystal monitor (FTM4). Additionally more accurate thickness values were obtained interferometrically by multiple - beam Fizeau frings method. A film thickness of $0.90 \mu \mathrm{m}$ was considered. The amorphous nature of the films was checked out by X-ray diffraction (XRD) analysis. The observed diffractogram showed only the broad hump that characterizes the amorphous materials, without any indication for even very weak peaks.

\subsection{Film Characterization}

\subsubsection{Optical Studies}

Optical transmission and reflection of the films was measured using double beam Jasco- V-570: UV-VIS-NIR spectrophotometer at normal incidence in the range $300-1350 \mathrm{~nm}$ with an accuracy of 0.25 and $0.5 \%$ respectively. In the present study the absorption coefficient $\alpha$ is calculated using the equation:

$$
\alpha=1 / \mathrm{d}\left[\ln \left(\frac{(1-R)}{T}\right)\right]
$$

where $\mathrm{d}$ is the film thickness and $\mathrm{R}, \mathrm{T}$ the reflectance and transmittance, respectively.

A transmission spectrum was also performed using AD110 photomultiplier Amplifier in the spectral range 300 to $1200 \mathrm{~nm}$. 


\subsubsection{Conductivity and Photoconductivity Measurements}

The hot point probe method was used to determine whether the semiconductor is $\mathrm{N}$ - or $\mathrm{P}$ - type. The potential difference when measured between the hot and the cold contacts (thermal Seeback effect) and the centerzero meter deflection identify the carrier type. The cold probe polarity therefore indicates the conductivity type.

Electrical conductivity of films was measured using the dc two- point probe method. Front contacts (planar gap-cell) were made of aluminum deposited by thermal evaporation at a pressure of $\sim 10^{-4} \mathrm{~Pa}$. The $10 \mathrm{~mm}$ long contacts were separated by $1 \mathrm{~mm}$ gap spacing. Silver conducting paste was used to ensure good Ohmic contacts which checked by measuring the currentvoltage characteristics. Keithley $(617 \& 238)$ meters were used to measure the bias voltage and the dc current, respectively while the temperature was adapted using an Oxford CDTC2 temperature controller.

The photoconductivity spectral response was measured using computerized CM110 1/8 monochromator $(0.1 \mathrm{~mm}$ slit). The light source was halogen lamp $\left(56 \mathrm{~mW} / \mathrm{m}^{2}\right)$ focused onto the sample through an optical system consisting of two convex lenses. The decay kinetics of the charge carriers was studied through measuring the ac photoconductivity in which the photocurrent is studied through the frequency modulation of the incident light. For this a mechanical chopper Stanford SR540 which gives symmetric square light pulses was used. The variation due to the modulation photocurrent was measured through lock in amplifier Stanford SR510. Thus a high sensitivity and noise suppression are achieved. Measurements were carried out at room temperature unless stated.

Dc conductivity and photoconductivity were measured in vacuum cryostat of $\sim 10^{-3} \mathrm{~Pa}$ to avoid sample oxidation in the high temperature range and prevent water vapor condensation in the low temperature range. Measurements were done over a wide temperature range extends from $100 \mathrm{~K}$ up to $325 \mathrm{~K}$.

\subsubsection{Photoinduced Changes}

For photoinducd observations the light exposure was performed by the halogen lamp for 180 minutes. After irradiation and almost under the same conditions the measured properties were again investigated. It has to be pointed out that the transmission spectra using the photomultiplier and the spectral dependence of the photoconduction were measured just after cessation of irradiation while transmission and reflection using spectrophotometer were measured few days latter. In fact this was not intended but as the work went on it was found interesting. 


\section{Results and Discussions:}

\subsection{Optical Absorption}

Above the exponential tail, the absorption coefficient of amorphous semiconductors has been observed to follow the relation:

$$
(\alpha h v)=A\left\{h v-E_{g}\right\}^{n},
$$

where $h v$ is the effective photon energy, $E_{g}$ is the optical band gap, A is constant and $\mathrm{n}$ an index have the values of 1/2,3/2, 2 and 3 depending on the nature of electronic transition and on localized state density-energy dependence. In present work, the calculated $\alpha$ value for $\mathrm{As}_{2} \mathrm{~S}_{3}$ film of thickness $0.9 \mu \mathrm{m}$ was found to be in the range $10^{4} \mathrm{~cm}^{-1}$. The usual method to determine the $E_{g}$ value involves plotting a graph of $(\alpha h v)^{1 / n}$ versus $h v$.

Non-direct optical transitions ( $\alpha h v)^{1 / 2}$ vs $h v$, observed in most amorphous materials, is not well obeyed in the present study. This is ascribed to the thermal history of the films. Annealing of the films at $443 \mathrm{~K}$ and for five hours decreases the degree of disorder and thereby the defects in the amorphous structure [20]. This may results in other transitions such as direct forbidden transitions $(n=3 / 2)$, which have been reported for some amorphous materials [21].

Figure (1) illustrates the experimentally obtained relation of $(\alpha \mathrm{h} v)^{2 / 3}$ versus photon energy $h v$. At higher absorption region, a least-square fit to the linear relationship is obtained. The optical gap energy $\mathrm{E}_{\mathrm{g}}$, determined by extrapolating the straight line portion to the energy axis, at the zero absorption coefficients, was found to be $1.9 \mathrm{eV}$, which is relatively small compared to the values previously reported [22]. The presence of a high concentration of localized states would produce absorption at energies less than the band gap and thereby is responsible for such low values. The weak absorption region at lower energy side is attributed to the presence of intraband transitions at localized states in the gap.

It is clearly observed Fig.(1) that the optical gap is lowered to $1.84 \mathrm{eV}$ after prolonged illumination, i.e. the well known effect of photodarkening [23]. As seen also from Fig.(2), as a result of illumination, the transmission edge shifts to longer wavelengths which confirm the photodarkening. The red shift manifests the fact that Urbach tail light can generate mobile carriers [24], holes in present $\mathrm{As}_{2} \mathrm{~S}_{3}$ films. 


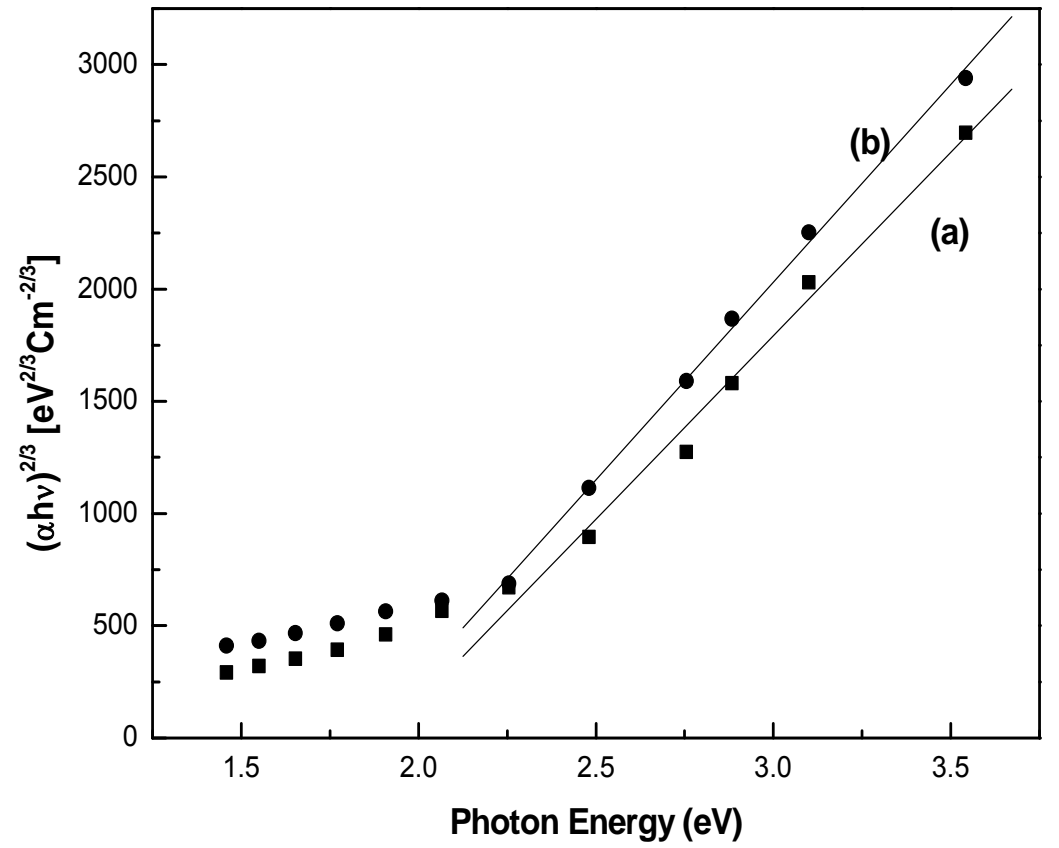

Fig. (1): Plot of $(\alpha h v)^{2 / 3}$ versus hv for film of thickness $0.90 \mu \mathrm{m}$ deposited at $305 \mathrm{~K}$ : a) before and b) after irradiation.

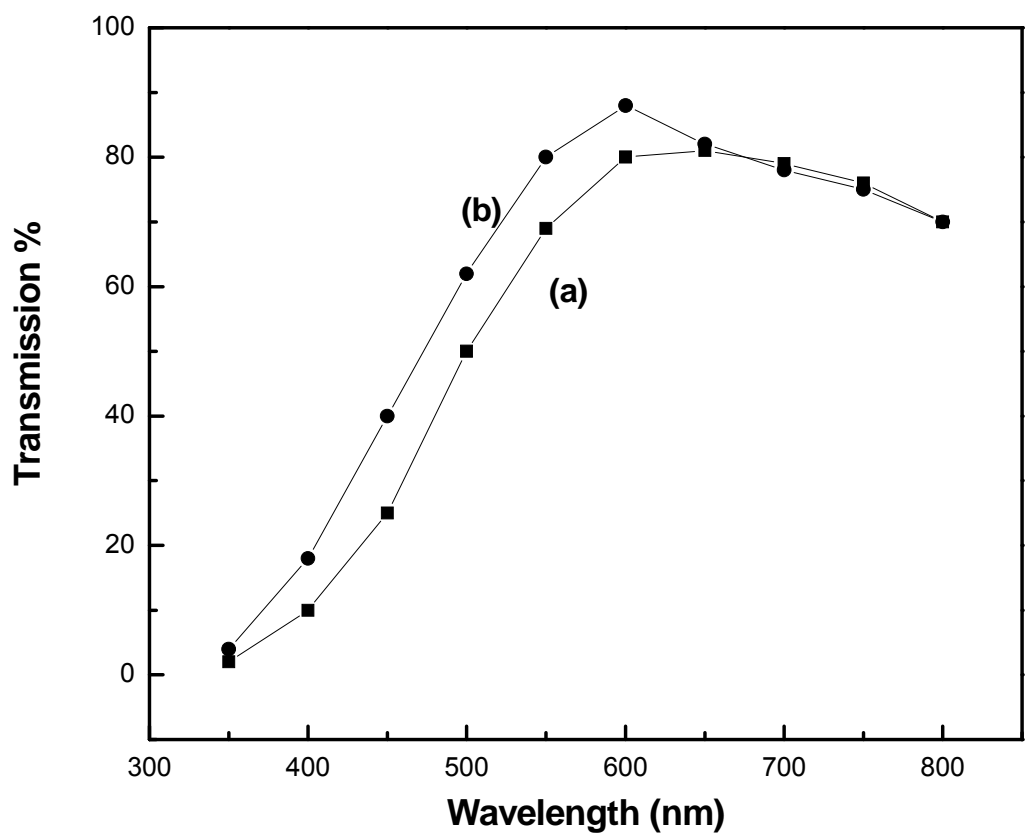

Fig. (2): Plot of tansmission versus wavelength: a) before and b) after irradiation. 
On the other hand one can see that the lines shape before and after irradiation are similar. This seems to be inconsistent with the above mentioned models of photodarkening that involve structural changes which would reproduce marked change in the behaviour. Hence the photodarkening is assumed to be photochemical effect in which changes due to light exposure occur only in the energy levels of the chalcogens $\left(\mathrm{C}^{\circ}-\mathrm{C}^{-}+\mathrm{h}\right.$ and $\left.\mathrm{C}^{0}-\mathrm{C}^{+}+e\right)$. The accumulation of $\mathrm{C}^{-}$and $\mathrm{C}^{+}$near band edges would give the observed spread in tail states and in turn the decrease in the gap.

The observed shift in optical absorption, measured after five days indicates that photodarkening stays for long time. The shift $(0.06 \mathrm{eV})$, deduced from Fig.(1), is small compared to $(0.12 \mathrm{eV})$ observed in quick measured film using the photomultiplier, Fig.(2). This suggests that in addition to the transient part which stays only for short time, a metastable part also exists. The present experimental results can not distinguish exactly between these two components, since different techniques are used but presents evidence for two types of relaxations. The faster relaxation component may be described by a phononassisted hopping of charges through localized tail states while the slow component of the relaxation due to the presence of potential fluctuations [25].

\subsection{Transport Properties}

\subsubsection{Photocurrent Spectra}

Figure (3) illustrates the spectral dependence of the photocurrent with its peak near $2.8 \mathrm{eV}$. The photocurrent is taken as the net photocurrent, i.e. the dc value subtracted from the total current. The optical band gap (at half maximum as shown by the arrow) was found to be $2.5 \mathrm{eV}$.

As can be seen, a small decrease of the photocurrent at $\mathrm{h} v>2.8 \mathrm{eV}$ may be due to one or more of the following reasons: (i) Inelastic collisions as a function of the kinetic energy of carriers and the surface recombination, a process by which the resultant potential energy of the surface defects is lowered. (ii) The unsaturated bonds present at the film surface which act as traps for photogenerated carriers. (iii) The moderate bulk recombination velocity compared to that at the film surface. The reduction at $\mathrm{h} v<2.4 \mathrm{eV}$ seems to reflect the decrease in absorption coefficient [26].

\subsubsection{Conductivity - Temperature Dependence}

Figure (4) shows the temperature dependence of dc conductivity $\left(\sigma_{\mathrm{dc}}\right)$ and steady state photoconductivity $\left(\sigma_{\mathrm{ph}}\right)$. In the measured range of temperature $(100-325 \mathrm{~K})$, the conductivities are proportional to the absolute temperature $\mathrm{T}$ and obey Arrhenius equation: 


$$
\sigma=\sigma_{0} \exp \{-\Delta \mathrm{E} / \mathrm{KT}\},
$$

where $\sigma$ is the conductivity at temperature $\mathrm{T} ; \sigma_{0}$ is a constant; $\Delta \mathrm{E}$ is the thermal activation energy and $\mathrm{K}$ is Boltzmann constant. As shown after illumination, the same dependence is obeyed.

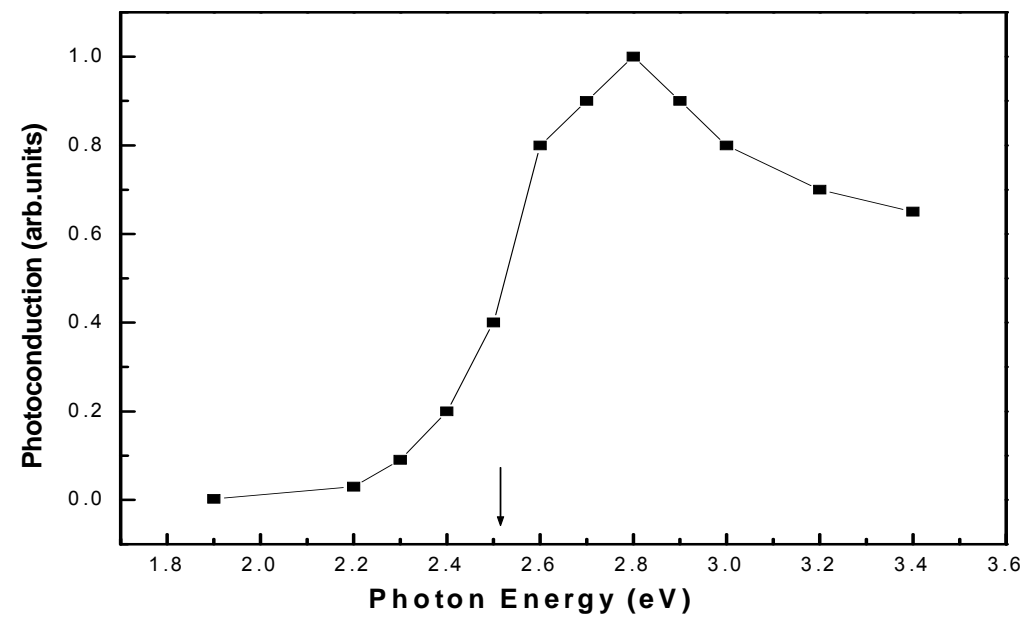

Fig. (3): Photon energy dependence of photocurrent for the as deposited film exposed to $56 \mathrm{Mw} / \mathrm{m}^{2}$.

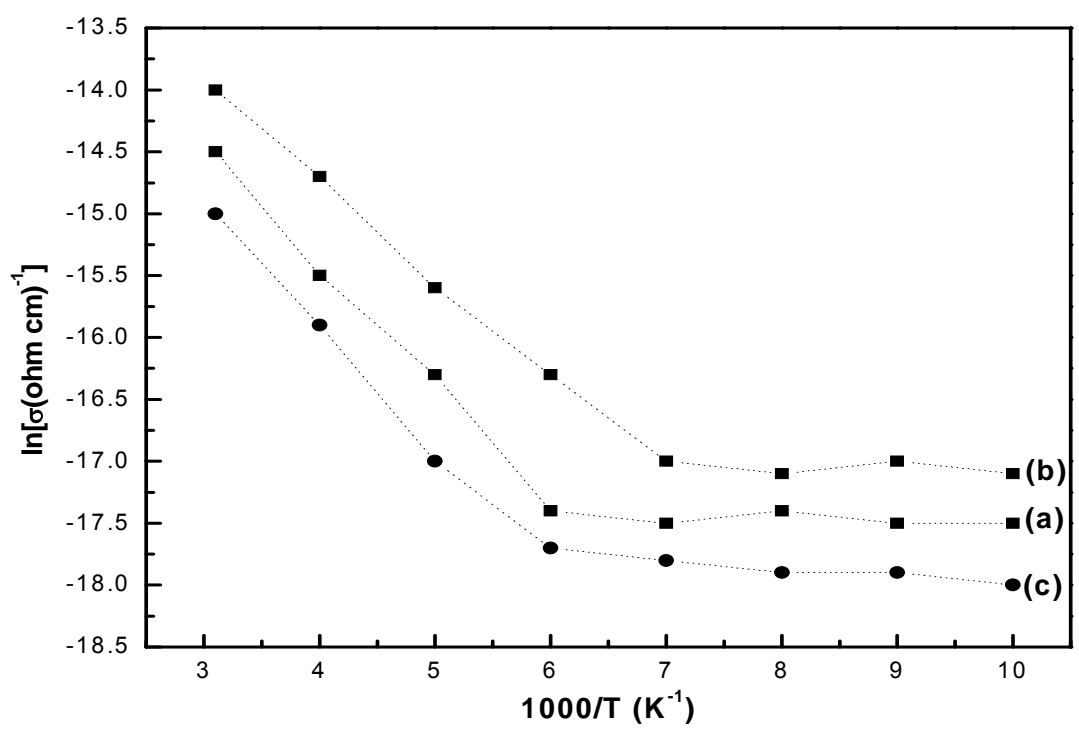

Fig. (4): The variations of the conductivity with temperature.

a) dark, b) Photoconductivity and c) Photoconductivity after illumination.

The $\ln \sigma-1 / \mathrm{T}$ plot shows two linear regions intersecting at a kink temperature $(150 \mathrm{~K})$. It is certain that different transport mechanisms operating in the higher $(\mathrm{T}>150 \mathrm{~K})$ and the lower $(\mathrm{T}<150 \mathrm{~K})$ temperature regions and are 
responsible for the observation of the kink in the plot. In the low temperature region the dominant carrier transport mechanism will be tunneling which is reflected by an almost temperature independent variation of the conductivity. It can be estimated that in this temperature range, the contribution from phonon scattering becomes insignificant compared with that caused by grain boundary defect states. In the temperature range $150-305 \mathrm{~K}$, the strong conductivity dependence indicates that the conduction is through an activated process.

The values of activation energies, calculated from the slope of the plot are found $0.80 ; 0.72$ and $0.71 \mathrm{eV}$ for dark; photoconductivity and photoconductivity after illumination, respectively. As seen the activation energy is reduced by irradiation. The thermal activation energy $\Delta \mathrm{E}$ is found lower than $1 / 2 \mathrm{E}_{\mathrm{g}}$ value (determined by optical methods) indicating that the Fermi position does not occur in the middle of the gap due to difference in smearing of valence and conduction bands in amorphous materials. The activation energy in the higher temperature region should be equal to the grain boundary height when the flow of charge carrier over the barrier is due to thermoionic process.

The similarity in the temperature dependence of both dark and photoconductivity indicates that the transport process is governed by the same carriers. The investigated films are found P-type semiconductors (as checked by the hot probe method) and the holes are then the main free carriers. As can be seen, prolonged exposure decreases the photocurrent (to be lower than its original value) indicating that the new metastable defects produced by illumination works as scattering (or recombination) centers, and thereby decrease the mobility (or carrier concentration). Hence this can be related to the limited effective carrier mobility and enhanced trapping because of these created defects. These defect sites can also cause shift below (or above) in the Fermi energy, where the majority defects are charged instead of being neutral at its original position. The observed reduction in activation energy can then be understood.

\subsubsection{Photoconductivity Kinetics}

In this the photoconductivity signal $\Delta \sigma / \Delta \sigma_{0}$ is plotted as a function of chopping frequency. $\Delta \sigma$ and $\Delta \sigma_{\mathrm{o}}$ are the ac and steady state components. The general feature of curves showed non-exponential decrease with the increase in frequency. The decay is more noticeable in the low frequency range. Fig.(5) illustrates the curves for as deposited and illuminated films, plotted in log scale under the same light intensity. As observed the logarithmic shape is not Gaussian and the dispersion, from ideal decay, increased a little by illumination. This explains the effect of defects in slowing the trapping processes. Multiple trapping in which the trapped carriers appear again by thermal reemission, is 
repeated many times till excitation disappears by interaction with recombination centers. The behaviors indicate that the recombination mechanisms before and after illumination are essentially the same. This suggests that the new created localized states are the same centers which dominate recombination process for the original states.

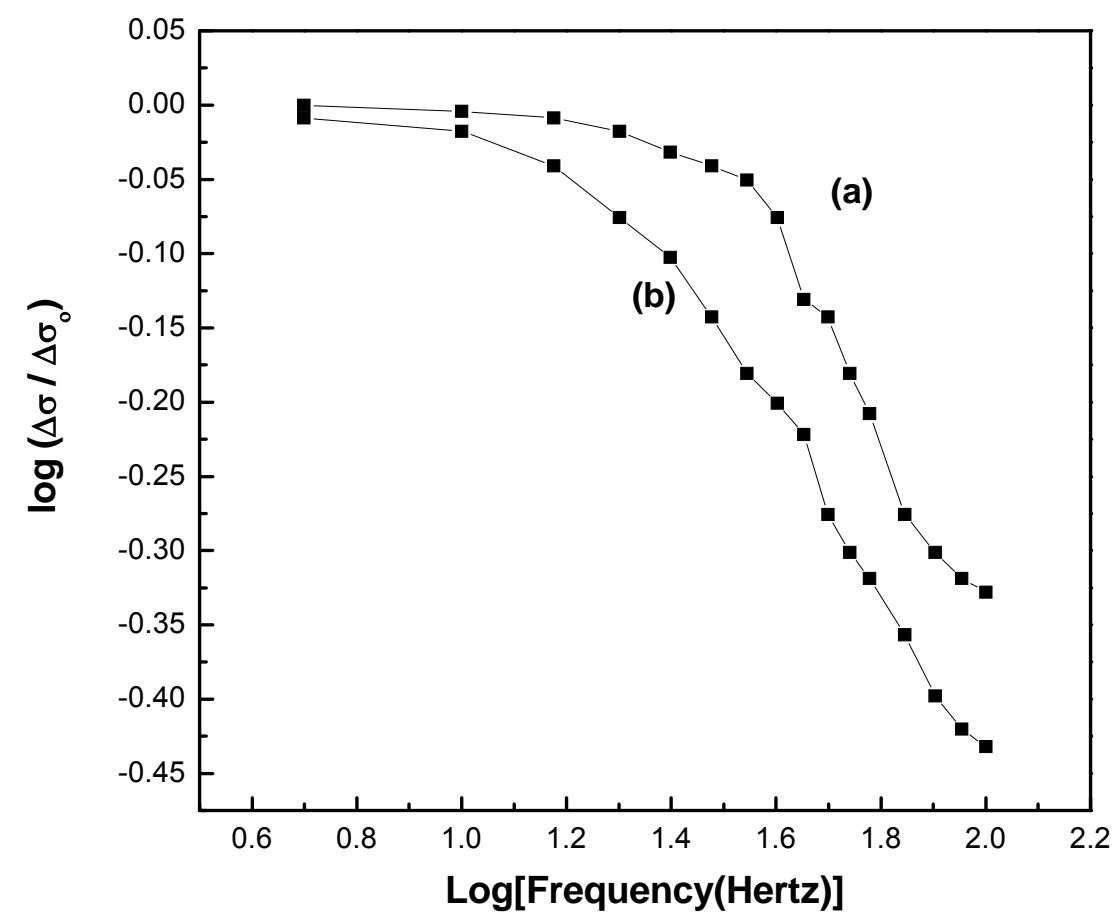

Fig. (5): Logarithmic plot of photoconductivity vs. frequency:

a) before and b) after irradiation.

\section{Conclusions:}

The properties of evaporated $\mathrm{As}_{2} \mathrm{~S}_{3}$ thin films (optical absorption, dc conductivity and photoconductivity) are compared before and after $3 \mathrm{~h}$ continuous irradiation. They exhibited the followings:

- The shift in optical absorption edge to smaller energies demonstrates the photodarkening in such films.

- After cessation of illumination, the photoinduced changes constitute both transient and metastable effects.

- By light exposure, the magnitude of photoconductivity decreases while the shape does not change, which predicts that the defect states created by 
illumination, dominate the recombination process in a similar manner to the original states.

- Dc conductivity and photoconductivity measurements exhibited two transport mechanisms at low and high temperature regimes. The similarity indicates that the transport is governed by the same carriers.

- The Similarity in behaviours of all the measured properties suggests that light induced changes could not be structural but photochemical ones.

\section{References:}

1. P. C. Taylor, P. Hari, A. Kleinhammes, P. L. Kuhns, W. G. Moulton and N.S. Sullivan, J.Non-Cryst.Solids 227-230, 770 (1998).

2. K. Tanaka, Y. Ohtsuka, Thin Solid films 57, 59 (1979).

3. S. D. Sartale, C. D. Lokhande, Materials Research Bulletin 35 1345, (2000).

4. J. Singh, K. Shimakawa "Advances in Amorphous Semiconductors" Taylor \& Francis, London (2003).

5. K. Tanaka, J. Opt. Adv. Mater. 3, 189 (2001).

6. K. Tanaka, S. Nakayama, J. Opt. Adv. Mater. 2, 5 (2000).

7. N. F. Mott, E. A. Davis "Electronic processes in Non-Crystalline Materials" Clarendon Press, Oxford (1979).

8. J. Tauc, R. Grigorovici, A. Vancu, phys. stat. sol. 15, 627 (1966).

9. S. K. Oleary, S. Zukotynski, J. M. Prez, Phys.Rev B 52, 7795 (1995).

10. H. Okamoto, K. Hattori, Y. Hamaka, J. Non- Cryst. Solids 198-200, 124 (1996).

11. H. Ticha, L. Tichy, P. Nagels, E. Sleeckx, R. Callaerts, J. of Phys. \& Chem. of Solids 61, 545 (2000).

12. P. Hari, T. Su, P. C. Taylor, P. L. Kuhns, W. G. Moulton, N. S. Sullivan, J.Non-Cryst. Solids 266-269, 929 (2000).

13. K. Tanaka, Phys.Rev.B 57, 5163 (1998).

14. A. Ganjoo, K. Shimakawa, K. Kitano, E. A. Davis, J. Non- Cryst. Solids 299-302, 917 (2002).

15. K. Shimakawa, N. Yoshida, A. Ganjoo, Y. Kuzukawa, J. Singh, Phil. Mag. Lett. 77, 153 (1998).

16. J. Dikova, K. Starbova, Vacuum 58, 490 (2000).

17. L. Tichy, H. Tcha, P. Nagels, R. Callaerts, Materials Lett. 36, 294 (1998).

18. G. Lucovsky and M. A. Paesler, phys. stat. sol. (C) 5, 1197 (2004).

19. A. Ganjoo, Y. Ikeda, K. Shimakaw, J. Non- Cryst. Solids 266-269, 919 (2000).

20. K. Tanaka, "Stracture and Excitaions in amorphous solids", ed. by G. Lucovsky and F. L. Galeener, American Institute of Physics, New York (1976). 
21. E. Abd El- Wahabb, M. M. El- Samanoudy, M. Fadel; Applied Surface Science 174, 106 (2001).

22. L. P. Deshmukh, J. S. Dargad, C. B. Rotti, Ind. J. Pure Appl. Phys. 33, 687 (1995).

23. V. Lyubin, M. Klebanov, B. Sfez, B. Ashkinadze; Materials letters 58, 1706 (2004).

24. H. Fritzsche, Phil. Mag. B 68, 561 (1993).

25. K. Morigaki, "Physics of Amorphous Semiconductors", Emperial College, London (1999).

26. K. Tanaka, J. Non. Crys. Solids 266-269, 889 (2000). 